\title{
Engineering
}

\section{Estimation of the physiological mechanical conditioning in vascular tissue engineering by a predictive fluid-structure interaction approach}

\section{Claudia Tresoldi, Elena Bianchi, Alessandro Filippo Pellegata, Gabriele Dubini \& Sara Mantero}

To cite this article: Claudia Tresoldi, Elena Bianchi, Alessandro Filippo Pellegata, Gabriele Dubini \& Sara Mantero (2017): Estimation of the physiological mechanical conditioning in vascular tissue engineering by a predictive fluid-structure interaction approach, Computer Methods in Biomechanics and Biomedical Engineering, DOI: 10.1080/10255842.2017.1332192

To link to this article: http://dx.doi.org/10.1080/10255842.2017.1332192

+ View supplementary material

Submit your article to this journal $\pi$
Published online: 01 Jun 2017. 


\title{
Estimation of the physiological mechanical conditioning in vascular tissue engineering by a predictive fluid-structure interaction approach
}

\author{
Claudia Tresoldi, Elena Bianchi, Alessandro Filippo Pellegata, Gabriele Dubini and Sara Mantero \\ Department of Chemistry, Materials, and Chemical Engineering 'Giulio Natta', Politecnico di Milano, Milan, Italy
}

\begin{abstract}
The in vitro replication of physiological mechanical conditioning through bioreactors plays a crucial role in the development of functional Small-Caliber Tissue-Engineered Blood Vessels. An in silico scaffold-specific model under pulsatile perfusion provided by a bioreactor was implemented using a fluid-structure interaction (FSI) approach for viscoelastic tubular scaffolds (e.g. decellularized swine arteries, DSA). Results of working pressures, circumferential deformations, and wall shear stress on DSA fell within the desired physiological range and indicated the ability of this model to correctly predict the mechanical conditioning acting on the cells-scaffold system. Consequently, the FSI model allowed us to a priori define the stimulation pattern, driving in vitro physiological maturation of scaffolds, especially with viscoelastic properties.
\end{abstract}

\section{ARTICLE HISTORY}

Received 23 October 2016

Accepted 15 May 2017

\section{KEYWORDS}

Small-caliber vessel tissue engineering; bioreactor; fluid-structure interaction model; mechanical conditioning; circumferential deformations; wall shear stress

\section{Introduction}

Nowadays, cardiovascular diseases represent one of the leading causes of death worldwide, especially in the high-income countries where these pathologies account for the highest mortality percentage (63\% of annual total deaths) (Alwan et al. 2011; WHO 2014).

In this context, vascular tissue engineering (VTE) is pivotal to produce functional non-immunogenic small-caliber $(<6 \mathrm{~mm})$ vessels, able to substitute pathological arterial tracts (Dermenoudis \& Missirlis 2010; Couet \& Mantovani 2012). To reach this goal, in vivo biochemical and biomechanical conditioning must be mimicked in vitro through pulsatile perfusion bioreactors (Schmedlen et al. 2003; Huang \& Niklason 2014; Tresoldi et al. 2015). They reproduce the physiological mechanical stimulation responsible for in vivo vascular homeostasis, due to the pulsatile blood flow as an effect of heart beats. When exposed to a working pressure in the range of $80-120 \mathrm{mmHg}$, vascular smooth muscle cells (VSMCs) are continuously subjected to cyclic strain $\left(\varepsilon_{\text {circ }}\right)$ about $10 \%$. Simultaneously, endothelial cells (ECs) and their progenitors (EPCs) are exposed to fluid wall shear stress (WSS) in the range of 5-20 dyne $/ \mathrm{cm}^{2}$ and of $0.1-2.5$ dyne $/ \mathrm{cm}^{2}$, respectively (Tresoldi et al. 2015). The mechanical conditioning is also responsible for VSMC circumferential disposition and for EC and EPC alignment along the flow direction (Huang \& Niklason 2014; Tresoldi et al.2015). WSS acts on ECs as a direct effect of flow, promoting EC-driven homeostasis, while $\varepsilon_{\text {circ }}$ keeps VSMCs to a contractile phenotype. Many studies in the literature highlighted that physiological pulsatile perfusion by means of bioreactors improves vascular development in terms of cell alignment and organization, phenotypic maintenance, extracellular matrix (ECM) production, and mechanical properties of the tissue-engineered vascular grafts (Hahn et al. 2007; Jeong et al. 2007; Seliktar et al. 2000; Zhang et al. 2009; Song et al. 2011; Rotenberg et al. 2012). Moreover, incremental mechanical stimulations enhance cell retention and adhesion to the scaffold, as well as cell proliferation, according to the fetal development approach (Couet et al. 2012; Tresoldi et al. 2015).

The control of mechanical conditioning, in particular WSS and $\varepsilon_{\text {circ }}$, acting on scaffolds and vascular cells is a key element to improve the correct in vitro functioning vascular development. Until now, many attempts have been made in VTE to control mechanical stresses on scaffold/vessel wall in perfusion bioreactors by means of a trial-and-errors process and by literature selection of mechanical stimulations patterns leading to a posteriori positive cellular outcomes (Hahn et al. 2007; Song et al. 2011; Patrachari et al. 2012; Gong et al. 2014).

The rigid wall theory (hereinafter called rigid model) is often used as the gold-standard in VTE to evaluate WSS,

CONTACT Claudia Tresoldi claudia.tresoldi@polimi.it

(6) Supplemental data for this article can be accessed at https://doi.org/10.1080/10255842.2017.1332192.

(c) 2017 Informa UK Limited, trading as Taylor \& Francis Group 
although it provides only a mean overestimated WSS value and neglects the important contribution of arterial scaffold compliance and the dynamics (Bertram 2009; Kanyanta et al. 2009; Boccafoschi et al. 2012; Reymond et al. 2013; Gong et al. 2014).

Considering these limitations, computational models have been used to improve the evaluation of stresses and deformations generated into tubular scaffolds while mechanically stimulated (Patrachari et al. 2012). For instance, computational fluid-dynamics (CFD), fluid-structure interaction (FSI), and finite element analysis (FEA) were used to predict WSS through scaffold pores while cells are proliferating (Cui et al. 2010; Lesman et al. 2010), to evaluate pulsatile flow of culture medium into a scaffold (Schmidt \& Tranquillo 2015), and to investigate scaffold wall stresses and deformations due to the fluid flow (Bilodeau et al. 2005; Zahedmanesh \& Lally 2012). In all the above cases, however, the 2-ways interaction between the fluid flow, that causes scaffold deformations and WSS, and the scaffold-cells systems, that respond to the fluid flow, is not considered (Bertram 2009). Despite the importance of the interaction between the fluid and the wall, FSI models were generally developed in the vascular field to predict fluid dynamics and solid stresses under mechanical properties modifications in aneurysms and stenotic vessels (Kelly \& O'Rourke 2010; Park et al. 2013).

Thus, in our work we will develop a scaffold-specific computational model using an FSI approach as a useful means for a priori evaluation of mechanical stimulations (WSS and $\varepsilon_{\text {circ }}$ ) acting on cells and tubular scaffolds, located into a pulsatile perfusion bioreactor for smallcaliber VTE. The FSI-based model, computed by COMSOL Multiphysics 4.4, will account for the viscoelastic mechanical properties of the scaffold, and will work under incremental flow rate, according to the fetal development approach. The aim of the study is to identify a priori a suitable pulsatile flow to apply to a perfusion bioreactor.

\section{Methods and materials}

Additional methods are described in the Supplementary Materials and Methods section and Supplementary Figures.

\subsection{Description of the experimental setup of the bioreactor}

The MiniBreath ${ }^{\odot}$ bioreactor (Harvard Apparatus Technology) (Figure 1(a) and (b)), hosting a tubular scaffold, was inserted in a hydraulic circuit able to produce a pulsatile flow rate.
This system was composed by a pulsatile pump (1405, Harvard Apparatus Technology), suppling the pulsatile flow to the tubular scaffold placed in the MiniBreath ${ }^{\circledast}$ bioreactor, by tuning the frequency $(\mathrm{f})$ and the stroke volume (SV). The hydraulic circuit was formed by silicone tubes $\left(\mathrm{PharMed}^{\odot}\right.$ ) and syringes (Figure 1) and included a resistance at the pump outlet, a resistance at the bioreactor inlet $\left(R_{\text {in }}\right)$, the block scaffold-bioreactor, a resistance in correspondence of the bioreactor outlet $\left(R_{c}\right)$, a compliance $\left(C_{c}\right)$, a downstream resistance $\left(R_{v}\right)$, and a free-air reservoir. The hydraulic circuit, described as an electric equivalent, was implemented in the computational model (Figure 1(c)).

\subsection{Description of the computational model}

The two-dimensional axial-symmetric FSI model represented the tubular construct in the MiniBreath ${ }^{\circledR}$ bioreactor, under the pulsatile perfusion provided by the hydraulic circuit described above.

\subsubsection{Computational domains and material properties}

The model consisted in two domains in the coordinate system defined by $r, \theta$, and $z$. The solid domain represented the tubular scaffold, described as cylindrical-shaped with length $l_{s}[\mathrm{~mm}]$, inner radius $r_{s}[\mathrm{~mm}]$, and wall thickness $s_{s}[\mathrm{~mm}]$. The material was characterized by the circumferential elastic modulus ( $E[\mathrm{MPa}])$ and shear relaxation modulus $(G(t)[\mathrm{MPa}]$, calculated using the Maxwell Generalized Model of Viscoelasticity) (Table 1). Mechanical properties for the Decellularized Swine Arteries (DSA), prepared as described by Pellegata et al. (2013), were obtained from ring uniaxial stretch and relaxation tests using MTS Synergie 200H (MTS Systems Corporation) (Pellegata et al. 2013). Regarding silicone and latex scaffolds, E was extracted from datasheet and $\mathrm{G}(\mathrm{t})$ was equal to $E / 2(1+v)$ (elastic material) (Fung 1993). Silicone conduits were used to develop the FSI model, while latex and DSA ones for its validation.

The fluid domain represented the volume within the scaffold, filled with the culture Dulbecco's Modified Eagle Medium, considered as isotropic, homogeneous, incompressible, and Newtonian, characterized by a fluid density $\left(\rho_{f}=1060 \mathrm{~kg} / \mathrm{m}^{3}\right)$ and a constant viscosity $\left(\mu_{f}=0.00082 \mathrm{~Pa}^{\star} \mathrm{s}\right)$ (Raimondi et al. 2002) (Figure 2).

Interaction between the two domains occurred by exchanging boundary velocity and fluid load at the fluid/ solid interface.

\subsubsection{Boundary conditions}

Solid domain: fixed radial edges of the scaffold were prescribed, reproducing the physical condition forced by the scaffold holders. The external lateral surface was free to 
(A)

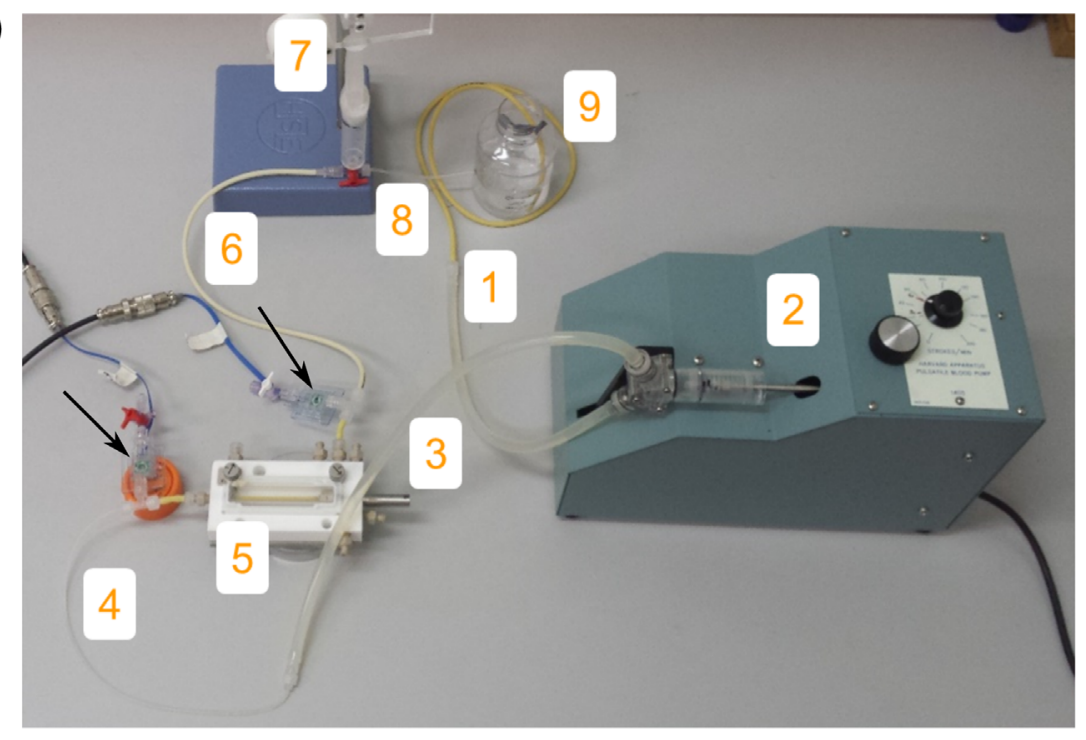

(B)
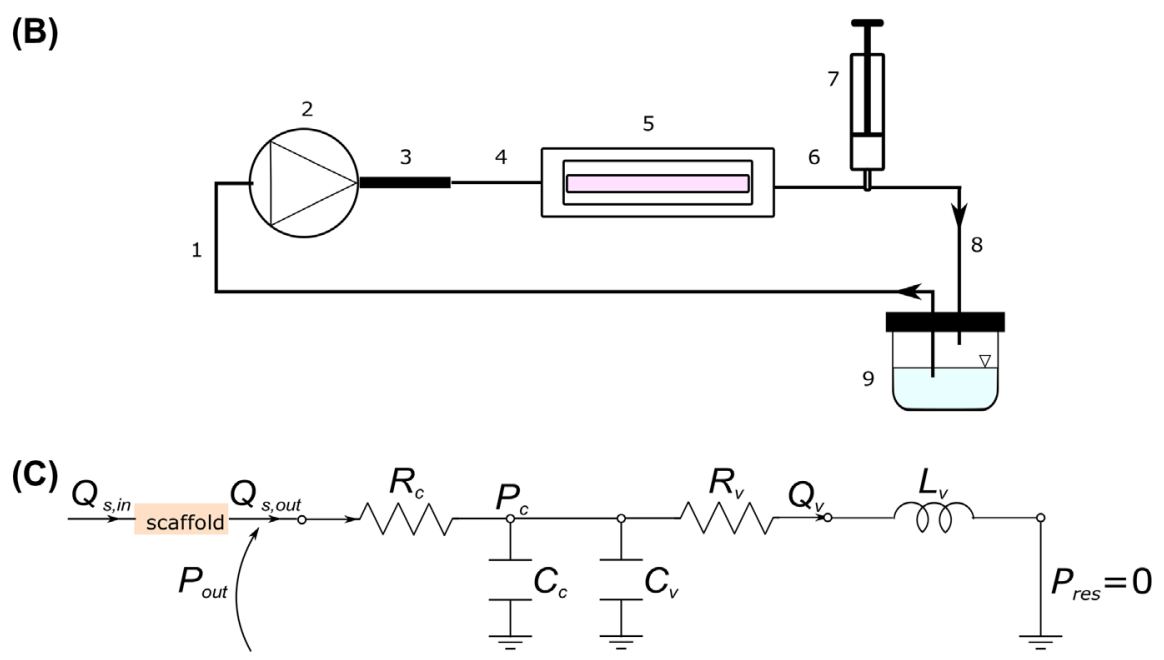

Figure 1. (a) Experimental set up and (b) representation of the main components of the hydraulic circuit for the pulsatile perfusion of a tubular scaffold. Arrows indicate the direction of the fluid flow. (1) resistance at the pump inlet $\left(R_{\text {in pump }}\right)$, (2) pulsatile pump (1405, Harvard Apparatus Technology), (3) resistance at the pump outlet $\left(R_{\text {out pump }}\right)$, (4) resistance at the bioreactor inlet $\left(R_{\text {in }}\right)$, $(5)$ the block scaffold-bioreactor, (6) resistance of the bioreactor outlet $\left(R_{c}\right),(7)$ compliance $\left(C_{c}\right)$, (8) downstream resistance $\left(R_{v}\right)$, and $(9)$ free-air reservoir. Black arrows indicate the pressure sensors (Edwards Lifescience LLC, Irvine, Canada, USA) for experimental pressure measurments. (c) Equivalent electric circuit of the hydraulic circuit of the system bioreactor-hydraulic circuit. $Q_{s, i n}$ is the flow rate entering the scaffold, $Q_{s, \text { out }}$ is the flow rate coming out the scaffold, and $P_{\text {out }}$ is the scaffold outlet pressure. The downstream tube is schematized with its compliance $\left(C_{v}\right)$, its resistance $\left(R_{v}\right)$, and its inductance $\left(L_{v}\right) . P_{c^{\prime}}$ and $Q_{v}$ are the pressure acting in correspondence of the compliance and the flow rate through the downstream tube, respectively. $P_{\text {res }}$ is the pressure in the free-air reservoir.

Table 1. Main parameters defining the solid domain for the tested scaffolds (Silicone, Latex, and DSA).

\begin{tabular}{|c|c|c|c|c|c|c|c|}
\hline \multirow[b]{2}{*}{ Scaffold } & \multicolumn{3}{|c|}{ Geometry } & \multirow{2}{*}{$\begin{array}{c}\text { Young's Modulus (E } \\
[\mathrm{MPa}])\end{array}$} & \multirow{2}{*}{$\begin{array}{c}\text { Shear Relaxation } \\
\text { Modulus (G(t) } \\
[\mathrm{MPa}])\end{array}$} & \multirow{2}{*}{$\begin{array}{l}\text { Poisson's modulus } \\
\text { (v) }\end{array}$} & \multirow[b]{2}{*}{ Density $\left(\rho\left[\mathrm{kg} / \mathrm{m}^{3}\right]\right)$} \\
\hline & $\mathrm{r}_{\mathrm{s}}[\mathrm{mm}]$ & $\mathrm{s}_{\mathrm{s}}[\mathrm{mm}]$ & $\mathrm{I}_{\mathrm{s}}[\mathrm{mm}]$ & & & & \\
\hline Silicone & 0.79 & 0.79 & 65.00 & 4.26 & - & 0.49 & $1.17 \cdot 10^{3}$ \\
\hline Latex & 1.00 & 1.00 & 65.00 & 1.39 & - & 0.49 & $0.92 \cdot 10^{3}$ \\
\hline $\begin{array}{l}\text { Decellularized swine } \\
\text { artery (DSA) }\end{array}$ & 1.50 & 0.615 & 50.00 & 0.40 & $\begin{array}{c}\mathrm{G}_{0}=0.117 \\
\mathrm{G}_{1}=0.009 \\
\tau_{1}=2.25 \cdot 10^{1} \mathrm{~s} \\
\mathrm{G}_{2}=0.109 \\
\tau_{2}=2.61 \cdot 10^{2} \mathrm{~s}\end{array}$ & 0.49 & $1.10 \cdot 10^{3}$ \\
\hline
\end{tabular}

move according to the fluid flow while a no-slip condition FSI interface was prescribed at the luminal surface (between the fluid domain and the solid one). An initial pre-stretch along $z$-axis $\left(\lambda_{z}\right)$ of $0.2 \mathrm{~mm}$ was prescribed in the solid domain, due to the initial pre-load of the scaffold when located into the bioreactor chamber (Figure 3(a)). 

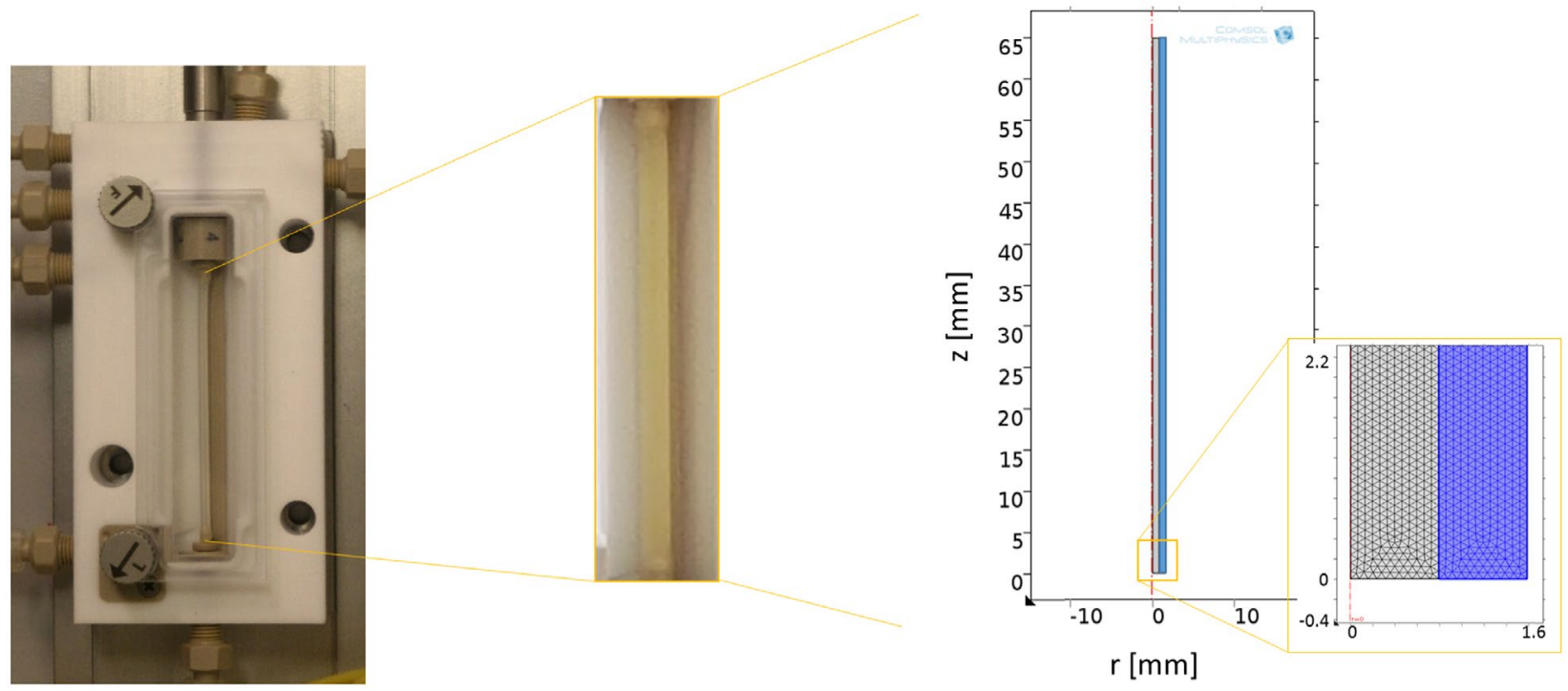

Figure 2. Domains of the FSI model. The solid domain is colored by blue and the fluid domain is in grey. In orange box, a detail of the two domains is shown, highlighting the mesh. The red dashed line indicates the axis of symmetry.

(A)

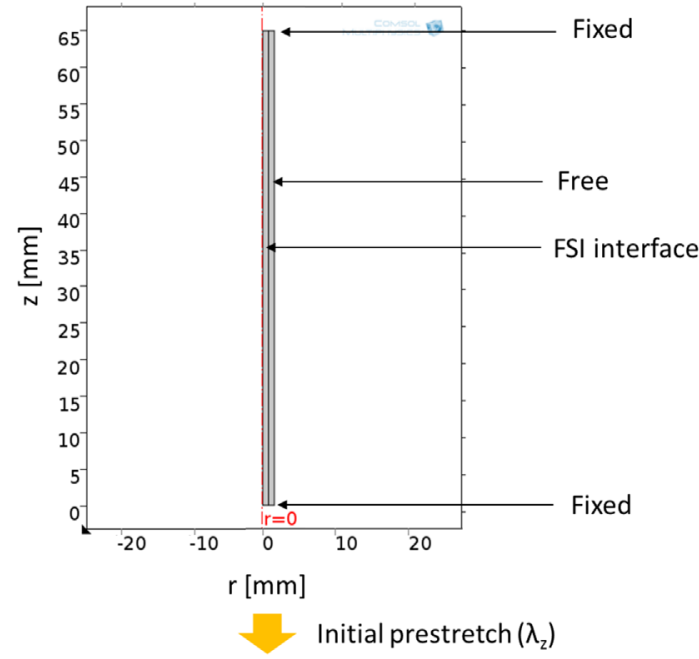

(B)

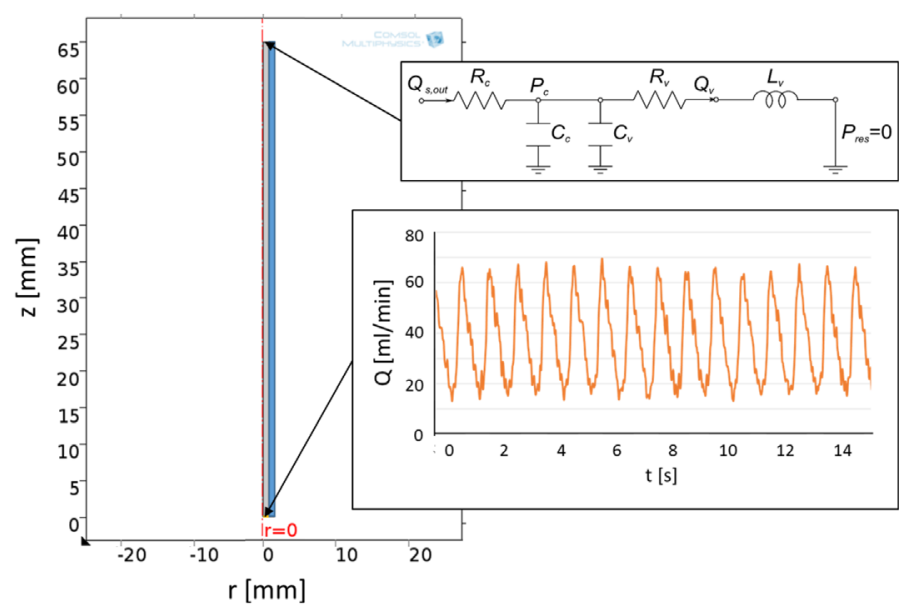

Figure 3. Boundary conditions of (a) solid domain, (b) fluid domain.

Fluid domain: a pulsatile and parabolic velocity profile (Figure 3(b)) was prescribed at the model inlet. The velocity curve $v_{\text {in }}(t)$ was obtained from the flow rate measured through Transonic ${ }^{\bullet} \mathrm{H} 110$ flowmeters (Transonic Systems Inc., USA), recorded by an acquisition shield (DAQPad 6020E, National Instrument, USA), and elaborated with LabVIEW 7.1 (National Instrument, USA).

The pressure $P_{s_{\text {out }}}$ at the interface between the FSI model and the lumped-parameter model was updated during the simulation and was considered spatially uniform. The pressure at the scaffold outlet was calculated by solving the system of ordinary differential equations describing the downstream lumped-parameter hydraulic circuit of the bioreactor whose parameters are experimental defined to ensure $80-120 \mathrm{mmHg}$ range for each flow rate (Figure $3(\mathrm{~b}))$. The pressure at the scaffold outlet depends on the measured flow rate out of the scaffold $\left(Q_{s_{\text {out }}}\right)$, considering the construct instantaneous deformations as functions of the scaffold viscoelastic mechanical properties. The pressure also included the contributes of the pressure drop caused by the outlet bioreactor geometry and by the luer-and-lock connections of the physical hydraulic circuit (Figure 1).

\subsubsection{Meshing}

The chosen mesh, defined according to an Arbitrary Lagrangian-Eulerian approach (Bavo et al. 2016), was triangular-shaped (Figure 2). Each triangular element had a 
minimum size of $1.630 \times 10^{-4} \mathrm{~mm}$ and a maximum size of $8.125 \times 10^{-2} \mathrm{~mm}$. The complete mesh consisted in 40,716 domains elements and 1902 boundary elements, obtained by a sensitivity analysis.

\subsubsection{Simulation}

The FSI model was solved using a time-dependent fully coupled solver, with a time stepping based on Backward Differentiation Formula (BDF) method. In each simulation 15 cycles were considered.

\subsubsection{Model results}

The following output variables were examined:

- pressures acting on the tubular scaffold, especially the pressure at the inlet, $P_{s_{\text {sin }}}$ and at the outlet, $P_{s_{\text {siu }}}$. $P_{s_{u}}$ corresponds to the value prescribed at the model outlet.

- circumferential deformation of the solid domain, $\varepsilon_{\text {circ }}$ due to the pulsatile medium flow. It represents the $\varphi \varphi$-direction elastic strain tensor solved during the simulation.

- wall shear stress, WSS, due to the medium velocity at the FSI interface, calculated according Equation 1:

$$
\mathrm{WSS}=\dot{\gamma} \cdot \mu_{\text {fluid }}
$$

where $\dot{\gamma}$ is the instantaneous shear rate calculated by COMSOL 4.4 solver and $\mu_{\text {fluid }}$ is the fluid viscosity.

\subsection{Validation of the viscoelastic FSI model}

To test the robustness of FSI model, we developed the model using conduits made of different materials (silicone, latex, and DSA). The validation of the viscoelastic FSI model was performed by evaluating the pressure acting on two different conduits (latex and DSA) when reducing progressively the elastic modulus of the chosen materials (Table 1). We validated the hydraulic circuits by setting the frequency to $60 \mathrm{bpm}$ and using SVs of $0.5,0.75$, $1,1.25$, and $1.5 \mathrm{ml}$ for latex scaffolds $\left(\varphi_{\text {int }}=2 \mathrm{~mm}, \varphi_{\text {ext }}=\right.$ $4 \mathrm{~mm}, \mathrm{l}=65 \mathrm{~mm}$ ), while we selected SV $=1 \mathrm{ml}$ for DSA scaffold $\left(\varphi_{\text {int }}=3 \mathrm{~mm}, \varphi_{\text {ext }}=4.23 \mathrm{~mm}, \mathrm{l}=50 \mathrm{~mm}\right.$ ), chosen as the reference circuit (Reymond et al. 2013).

Each hydraulic circuit was experimentally tuned to obtain a pressure range of $80-120 \mathrm{mmHg}$ in the scaffold. The same output variables were evaluated. In addition, $\varepsilon_{\text {circ }}$ was experimentally evaluated in the DSA scaffold only (paragraph 4).

\subsection{Comparison with the analytical models}

To reinforce the value of the implemented FSI model, the FSI results were compared with analytical models describing the same problem under two different conditions, the rigid model and the model described by the theory of thick wall for vessels.

\subsubsection{Rigid model}

The scaffold was considered as a rigid tubular construct. WSS were defined by the Hagen-Poiseuille equation:

$$
\mathrm{WSS}=\frac{4 \cdot \mu \cdot Q_{i n}(t)}{\pi \cdot r_{s}^{3}}
$$

where $Q_{i n}(t)$ is the flow rate recorded by flowmeters.

\subsubsection{Thick-wall model}

According to the theory of thick wall for the vessels (for linear elastic isotropic material) (Montevecchi \& Redaelli 2007), circumferential stresses, $\sigma_{\theta \theta}[\mathrm{MPa}]$ at the fluid/scaffold interface were calculated as follow:

$$
\sigma_{\vartheta \vartheta}=\frac{p(t) \cdot r_{s}^{2}}{\left(r_{s}+s_{s}\right)^{2}-r_{s}^{2}} \cdot\left(1+\frac{\left(r_{s}+s_{s}\right)^{2}}{r_{s}^{2}}\right)
$$

where $p(t)$ is the pressure recorded by sensors, $r_{s}$ is the scaffold radius in undeformed condition and $s_{s}$ is the scaffold wall thickness.

The circumferential deformations, $\varepsilon_{\theta \theta}[$ adim $]$, were calculated according to Equation (4):

$$
\varepsilon_{\theta \theta}=\frac{\sigma_{\vartheta \vartheta}}{E_{s}}
$$

where $E_{s}$ is the scaffold Young's modulus in $\theta \theta$-direction.

The WSS were calculated according to Equation (5):

$$
\mathrm{WSS}=\frac{4 \cdot \mu \cdot Q_{i n}(t)}{\pi \cdot r(t)^{3}}
$$

where $\mu$ is medium viscosity, $Q_{\text {in }}(t)$ is the flow rate recorded by flowmeters at the same moment of the pressure recording, and $r(t)$ is the radius in deformed configuration, equal to $r_{s} \cdot\left(1+\varepsilon_{\text {circ }}(t)\right)$.

\subsection{Results analyses}

\subsubsection{Pressure investigations}

The FSI-estimated pressure acting on the scaffold, $P_{\text {scaff }}$ $[\mathrm{mmHg}]$ was calculated as the mean value between the pressure at the inlet $P_{s_{\text {in }}}$ and at the outlet $P_{s_{\text {out }}}$ acting onto the tubular construct, considering negligible the pressure drop along the scaffold in comparison with the global working pressure of the bioreactor circuit (the maximum percentage difference between $P_{s_{\text {in }}}$ and $P_{s_{\text {out }}}$ was $5.93 \%)$. Moreover, the maintenance in phase of $P_{s_{\text {out }}}$ and $P_{s_{\text {out }}}$ allowed direct averaging of the two signals. A direct 
qualitative comparison of $P_{\text {scaff }}$ with the experimental pressure curve was performed for silicone, latex, and DSA scaffolds.

\subsubsection{Mechanical conditioning investigations}

To verify the mechanical conditioning acting on the vascular cells-scaffold system under pulsatile perfusion, $\varepsilon_{\text {circ }}$ and WSS estimated by the FSI model and calculated by the analytic model were analyzed for silicone, latex, DSA. Experimental values of $\varepsilon_{\text {circ }}$ in the biological scaffold were measured using the software ImageJ 1.410 (Wayne Rasband National Institutes of Health, USA), extracting frames from recorded videos at maximum and minimum pressure and considering the rest condition (circuit filled with culture medium but with the pump off) as the reference instant.

\subsubsection{Statistical analyses}

Statistical analyses were performed to compare $\varepsilon_{\text {circ,max }}$ and WSS $_{\max }$ predicted by analytic (rigid and thick-wall) and FSI models for all the considered conduits. For the DSA scaffold the comparison was extended to the experimental model and to operation under minimal mechanical conditioning $\left(\varepsilon_{\text {circ,min }}\right.$ and WSS min $\left._{\text {min }}\right) \cdot \varepsilon_{\text {circ,max }}, \varepsilon_{\text {circ,min }}$, WSS max $_{\text {, }}$ and WSS $_{\text {min }}$ were expressed as mean and standard deviation of at least ten peak values to take into account the variability of pulsatile peaks of $\varepsilon_{\text {circ }}(t)$ and WSS(t) due to the inlet real flow rate. One-way and multivariate analysis of variance (ANOVA) tests with Tuckey's multiple comparison correction were performed using GraphPad Prism software (GraphPad Software, Inc.). A significance level of $p$-value $<0.05$ was applied.

\section{Results}

\subsection{Pressure investigation}

The comparison between the experimental pressure $\left(P_{\text {scaff,exp }}\right)$ and the computed pressure $\left(P_{\text {scaff,FSI }}\right)$ showed that the FSI model nicely predicts the pressures acting on the tubular silicone scaffold into the range of $80-120 \mathrm{mmHg}$, with a minimum and maximum error in the FSI pressure estimation of $2 \%$ and $10 \%$, respectively (Figure 4 ). Dynamics of the pressure signals was correctly estimated for most of the considered SVs, except for the case with $\mathrm{SV}=1.25 \mathrm{ml}$. Furthermore, the simulated pressures resulted rather irregular due to the noise of the inlet flow rate input.

To validate the FSI model, we used it with latex and DSA scaffolds, by varying Young's modulus and the scaffold length.

$P_{\text {scaff }}$ was adequately estimated by the model for latex scaffold and DSA, as well as for silicone scaffold (Figure 5(a) and (b)). The FSI model correctly described the experimental setup in terms of working pressure range for all considered SVs, except for the cases of latex scaffold with $\mathrm{SV}=0.75 \mathrm{ml}$ and $\mathrm{SV}=1.25 \mathrm{ml}$.

\subsection{Mechanical conditioning investigations}

To evaluate the mechanical conditioning acting on cells in perfusion dynamic cultures, WSS and $\varepsilon_{\text {circ }}$ were analyzed as functions of the different mechanical properties (Young's modulus in $\theta \theta$-direction) of the scaffold

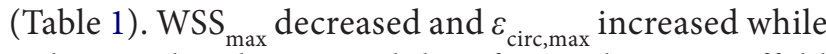
reducing the elastic modulus, from silicone scaffold to DSA (Figure 6). Moreover, $\varepsilon_{\text {circ }}$ and $\mathrm{WSS}_{\max }$ were uniform along the scaffold length, except at the constrained scaffold ends. In addition, $\varepsilon_{\text {circ }}$ were constant at varying flow rates, since the hydraulic circuits always operated in the same pressure range $(80-120 \mathrm{mmHg}$ ). On the contrary, WSS ${ }_{\max }$ increased with increasing flow rate.

With regard to the DSA scaffold, we performed experimental measures of $\varepsilon_{\text {circ }}$ at both maximum (120 $\mathrm{mmHg}$ ) and minimum $(80 \mathrm{mmHg})$ pressure values $\left(\varepsilon_{\text {circ,max }}\right.$ and $\varepsilon_{\text {circ,min }}$, respectively). These deformation values agreed with the circumferential deformations estimated by the viscoelastic FSI model (Figure 6(c)). The FSI-simulated values were statistically comparable to the experimental ones ( $p>0.05)$, both for $\varepsilon_{\text {circ,max }}$ and $\varepsilon_{\text {circ,min }}$ and completely fell within the experimental calculated range, with the maximum around $10 \%$. The computed WSS values in the scaffold, due to lower stiffness, fell within the range of 0.100-3.960 dyne $/ \mathrm{cm}^{2}$, also affecting the scaffold ends, where $\varepsilon_{\text {circ }}$ reached the minimum for the scaffold constraints (Figure 6(c)).

\subsection{Comparison with the analytical model}

When comparing the rigid model $\left(\varepsilon_{\text {circ }}\right.$ was 0 for definition) with the thick-wall and the FSI one (Figure 7(a) and (b)), mean $\varepsilon_{\text {circ,max }}$ resulted statistically different for silicone and latex scaffolds, as expected. Silicone $\varepsilon_{\text {circ, max }}$ from the thick-wall model was comparable with that from the FSI model. The two models were statistically different $(p<0.05)$ for latex, although $\varepsilon_{\text {circ,max }}$ was close to FSI numerical results. Moreover, the thick-wall and the FSI values accounted for scaffold deformations and pressure variability (Figures 4 and 5(a)). The WSS max $_{\text {values in the }}$ rigid model were significantly higher $(p<0.05)$ and were overestimated at all SVs. This behavior was not shown by the thick-wall model, which provided WSS ${ }_{\max }$ values closer to the FSI values for silicone and latex scaffolds (Figure 7(a) and (b)). The incremental trend was present in all models. 

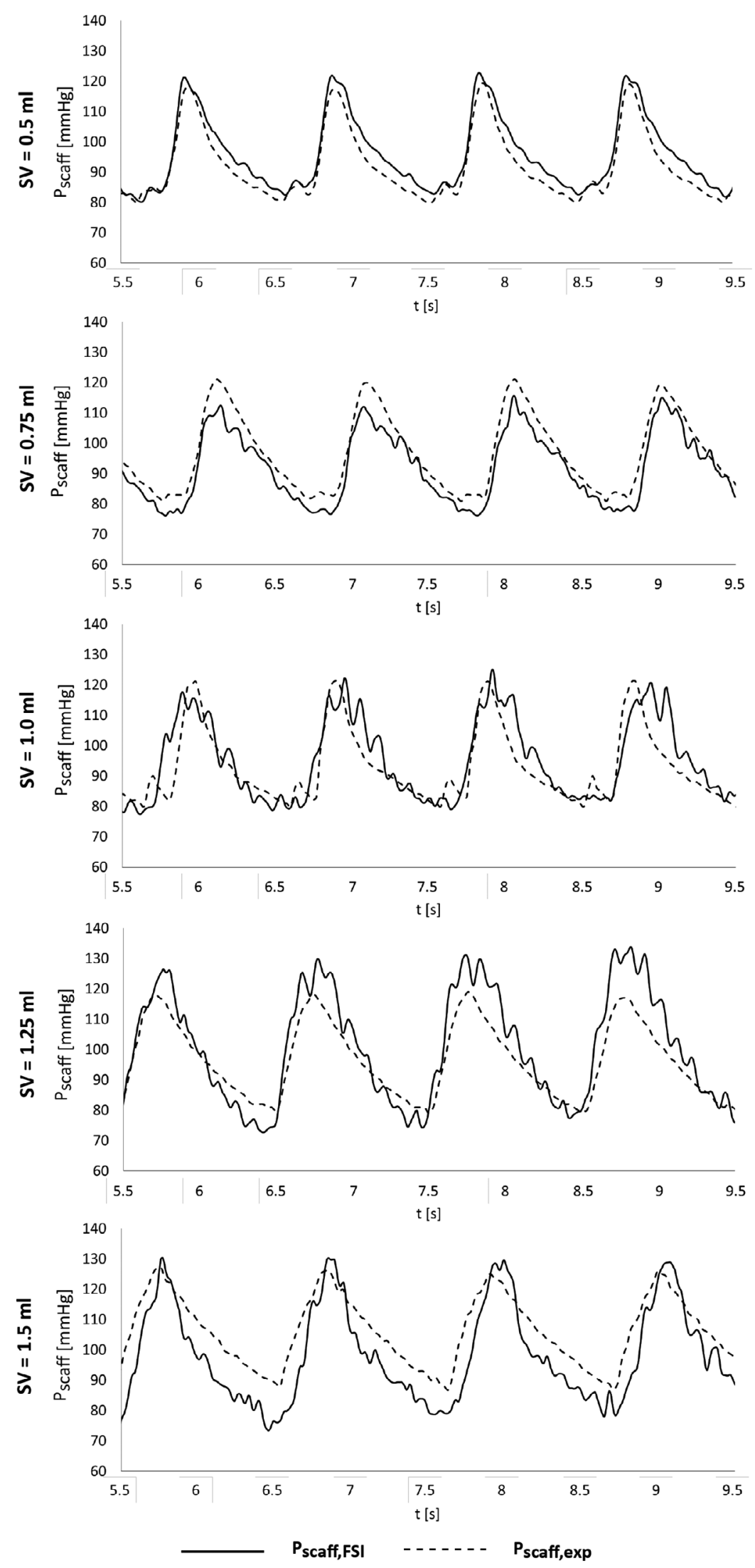

Figure 4. Pressure curves in correspondence of the silicone tubular scaffold. $P_{\text {scaff,Fsl }}$ is the model-estimated pressure and $P_{\text {scaffexp }}$ is the experimentally measured pressure. 

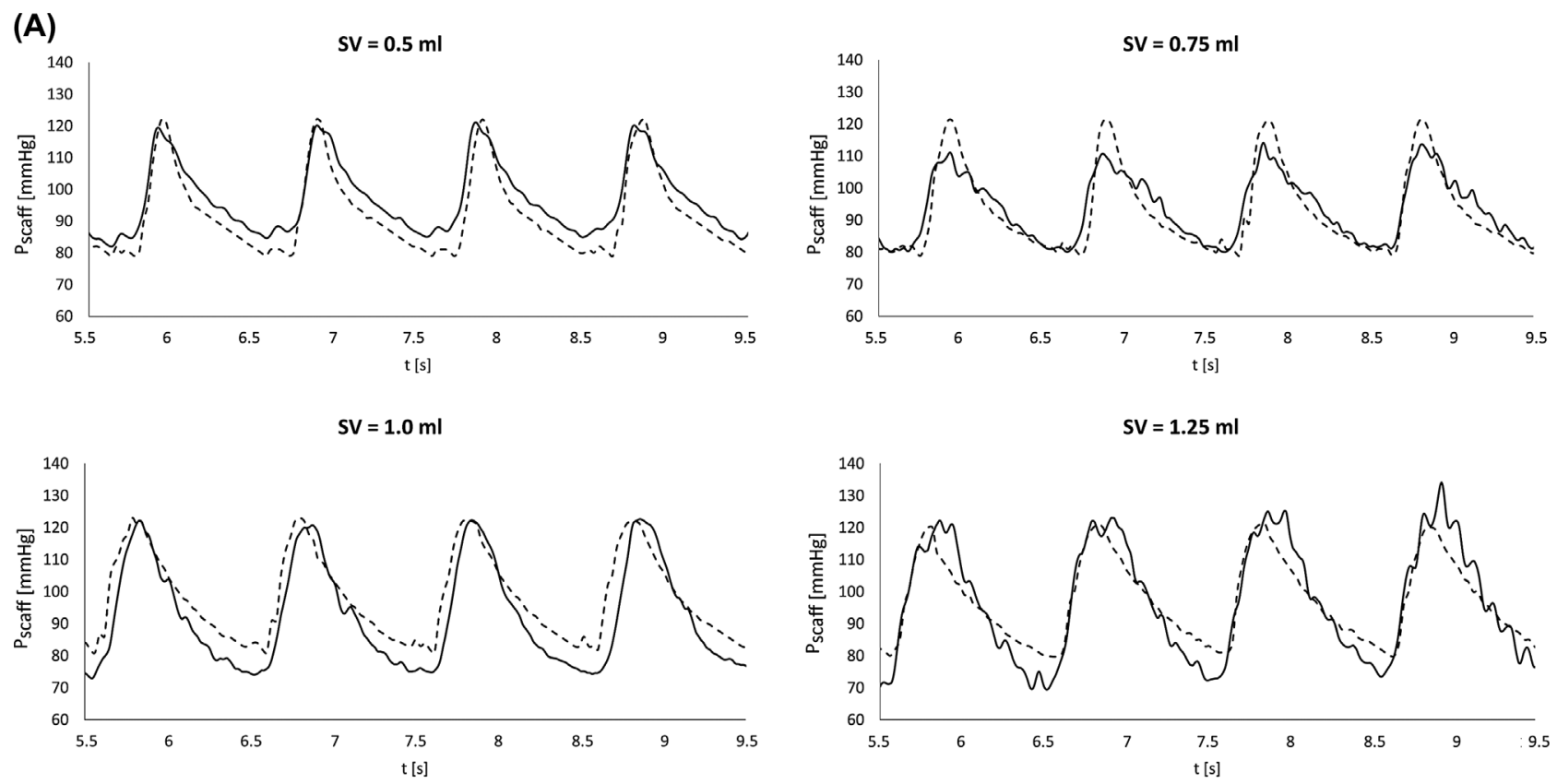

$\mathrm{SV}=1.5 \mathrm{ml}$

(B)
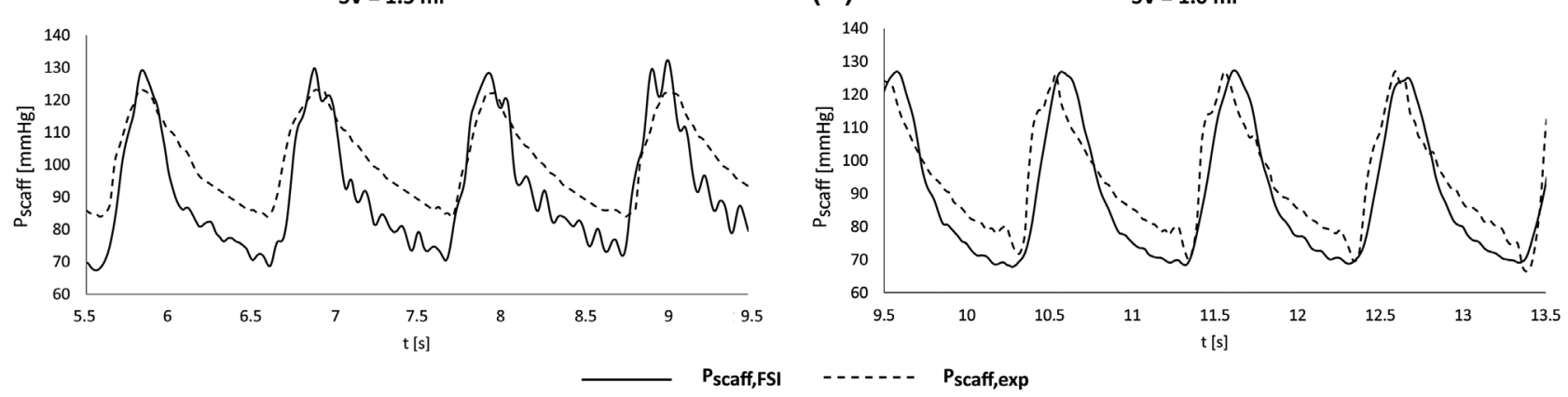

Figure 5. Pressure curves calculated as mean between scaffold inlet pressure and scaffold outlet pressure for (a) the latex tubular scaffold and (b) the DSA. $P_{\text {scaff,Fsl }}$ is the model-estimated pressure and $P_{\text {scaff,exp }}$ is the experimentally measured pressure.

(A)
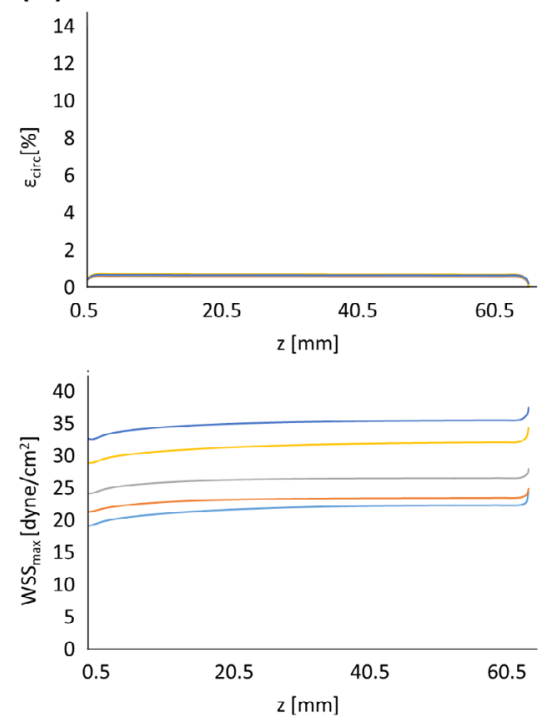

(B)
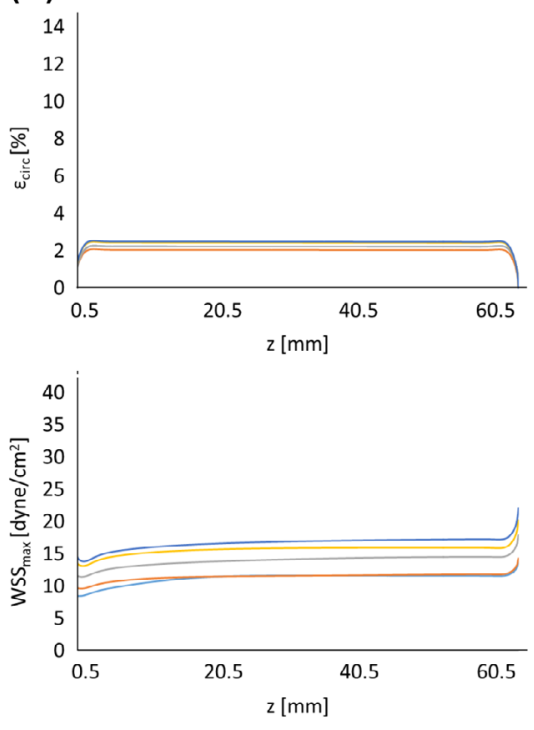

$-\mathrm{SV}=0.5 \mathrm{ml} \quad-\mathrm{SV}=0.75 \mathrm{ml}-\mathrm{SV}=1.0 \mathrm{ml} \quad-\mathrm{SV}=1.25 \mathrm{ml} \quad-\mathrm{SV}=1.5 \mathrm{ml}$
(C)
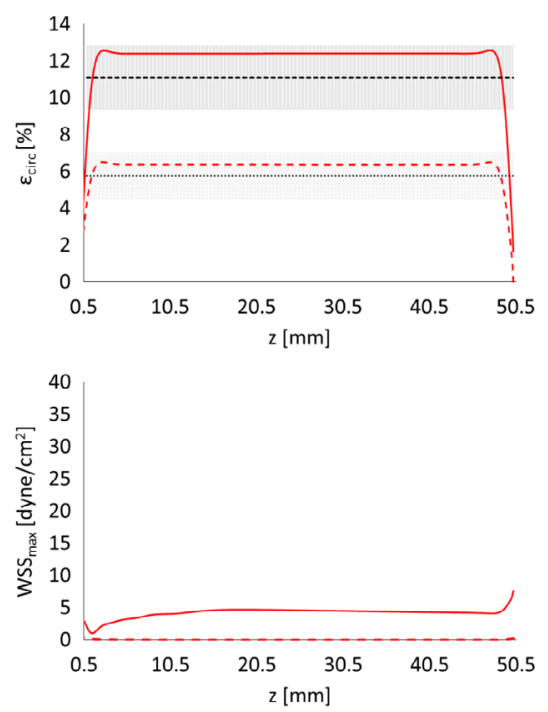

Figure 6. $\varepsilon_{\text {circmax }}$ and $W S S_{\text {max }}$ for (a) Silicone scaffold, (b) Latex scaffold, and (c) DSA, estimated by the FSI model. For DSA maximum (continued line) and minimum (dotted line) values are reported. 

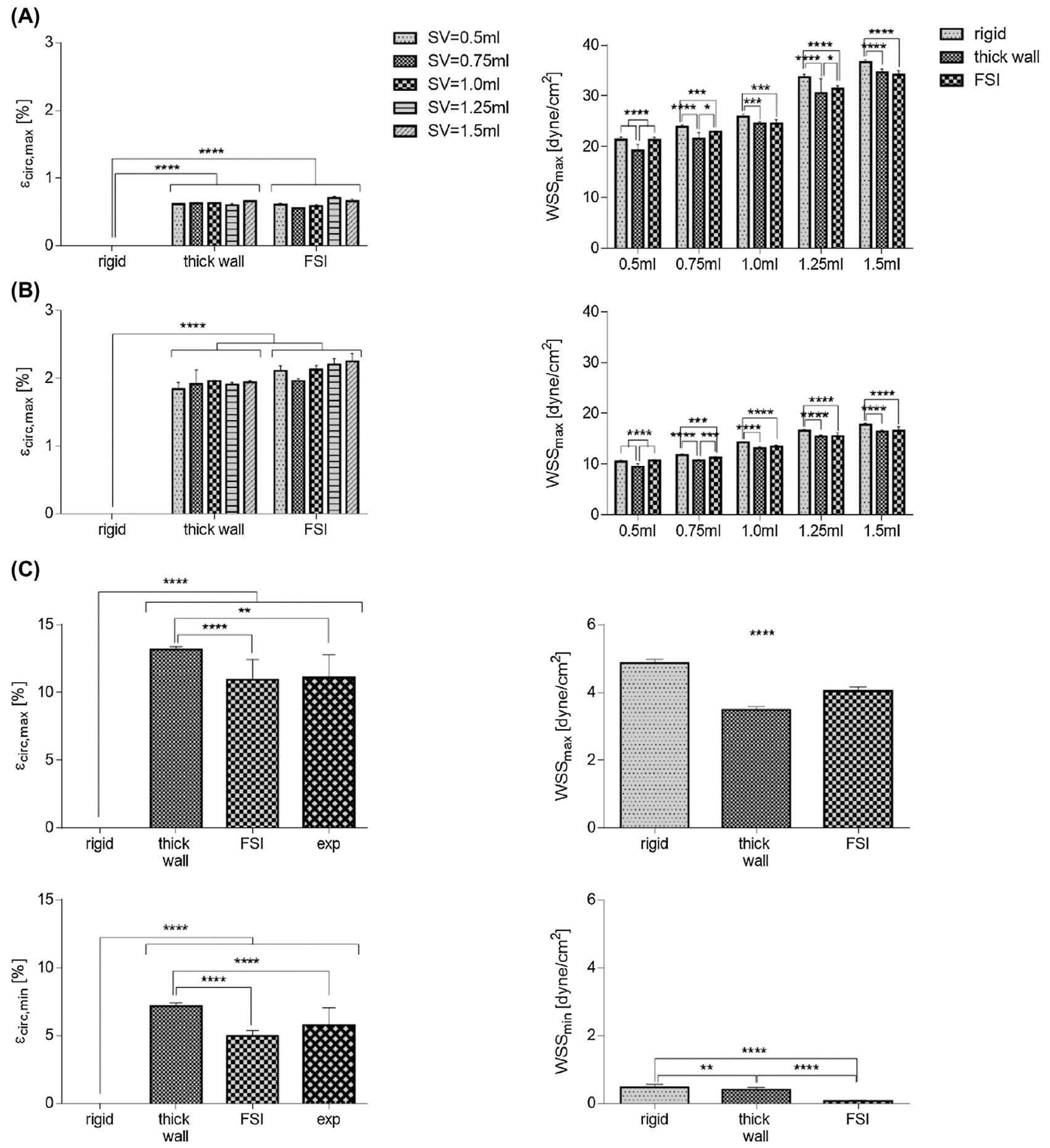

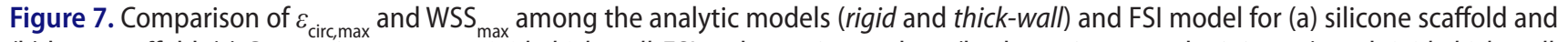
(b) latex scaffold. (c) Comparison among rigid, thick-wall, FSI and experimental $\varepsilon_{\text {circ }}$ (both maximum and minimum), and rigid, thick-wall, and FSI WSS (both maximum and minimum).

Regarding DSA (Figure 7(c)), the FSI-computed mean values of $\varepsilon_{\text {circ, } \max }$ and $\varepsilon_{\text {circ,min }}$ were compared with results from the two analytic models and the experimental values. Both the FSI-computed $\varepsilon_{\text {circ,max }}$ and $\varepsilon_{\text {circ,min }}$ were statistically comparable $(p>0.05)$ with the experimental ones. On the contrary, the rigid model significantly underestimated $(p<0.05) \varepsilon_{\text {circ,max }}$ and $\varepsilon_{\text {circ,min }}$ in DSA scaffold and the thick-wall model overestimated them in comparison to the FSI and the experimental $\varepsilon_{\text {circmax }}$ and $\varepsilon_{\text {circmin }}$, respectively. At the same time, WSS values calculated by the analytical models were significantly different from the FSI-computed ones. The rigid model overestimated $\mathrm{WSS}_{\max }$, as well as WSS $_{\text {min }}$, but the thick-wall model underestimated WSS ${ }_{\text {max }}$ as a consequence of the overestimation of $\varepsilon_{\text {circ,max }}$. 


\section{Discussions}

In this study, we investigated the ability of the FSI model to describe a perfusion bioreactor with its hydraulic circuit for Small-Caliber VTE and adequately identify the suitable pulsatile flow rate on the basis of an a priori evaluation of mechanical conditioning acting on the cells-scaffold system.

To reach this goal, we designed the hydraulic circuit to achieve a working pressure in the scaffold in the range of $80-120 \mathrm{mmHg}$, experimentally recorded by pressure sensors. The FSI model adequately predicted the working pressures of the system, since they were comparable with the experimental pressure. The FSI model worked well, independently from scaffold mechanical properties, as demonstrated by the three different scaffolds used, progressively reducing Young's modulus (from 4.26 MPa of silicone to $0.40 \mathrm{MPa}$ of DSA) (Figures 4 and 5). This evidence was mainly related to the choice of the combination of $R_{v}$ and $\mathrm{C}_{c}$. In fact, the minimum working pressure, completely independent from the scaffold, highly depended on the pressure drop due to $R_{v}$, and depended on the flow rate passing through the downstream hydraulic circuit. Furthermore, the FSI-computed pressures were affected by the inlet flow rate waveform, causing a mismatch in pressure dynamics and generating noisy signals due to the fluid oscillation detected by the flowmeters during the experiments.

The second part of this work consisted in the evaluation of the main mechanical stimulations ( $\varepsilon_{\text {circ }}$ and WSS) by means of the FSI model, required to generate a physiological vascular homeostasis. Circumferential strains, $\varepsilon_{\text {circ,max }}$ in silicone and latex scaffolds were quite constant for all the considered SVs (Figure 6(a) and (b)) since the bioreactor-scaffold system always operated at the same pressure $(80-120 \mathrm{mmHg}$ ), except for a small variability. For DSA, we performed also experimental measures of $\varepsilon_{\text {circ,min }}$ and $\varepsilon_{\text {circ,max }}$. The FSI-computed and experimental-calculated strain values were comparable between them, but statistically different when compared to the results provided by the two analytic models (Figure 6(c)). The $\varepsilon_{\text {circ }}$ overestimation, both for maximum and minimum values, produced by the thick-wall model could be due to the hypothesis of the scaffold being made of an elastic material, instead of being viscoelastic, as the FSI model correctly did. Interestingly, experimental $\varepsilon_{\text {circ,max }}$ resulted about $10 \%(11.580 \pm 0.605)$, that is the maximum physiological deformations which act on VSMCs in the presence of a blood perfusion of $80-120 \mathrm{mmHg}$ (Kona et al. 2009; Stoitsis et al. 2007). Instead, the thick-wall $\varepsilon_{\text {circ,- }}$ $\max (13.174 \pm 0.198)$, incomparable with the experimental values and closer to the lower limit for pathological VSMC phenotype (15\%), showed its limits for viscoelastic materials. These evidences confirmed the importance of viscoelastic properties of arteries which reduce wall displacement (Wang et al. 2015). In the arterial wall, the uncoiling and the scrolling of elastin and collagen fibers of the arterial ECM are responsible for the reduction of $\varepsilon_{\text {circ }}$ (Sokolis et al. 2006; Silver et al. 2001). The adopted FSI model correctly reproduced this behavior due to the constitutive equations of Maxwell model for viscoelastic materials. Consequently, in comparison to the rigid and the thick-wall theories, the FSI model correctly estimates the circumferential strains, and provides more reliable results as it includes both the compliance and the viscoelastic properties of the scaffold wall (Wang et al. 2015; Anayiotos et al. 1994).

The WSS analyses highlighted the strong relationship with Young's modulus and with circumferential strains. The direct comparison of WSS values in silicone, latex, and DSA scaffolds (Figure 6) showed the progressive WSS decrease at increasing $\varepsilon_{\text {circ }}$ and Young's modulus. Moreover, FSI-computed WSS of DSA resulted significantly smaller than those in the rigid model, while WSS ${ }_{\text {max }}$ from the thick-wall theory was lower than that from the FSI model. These evidences further confirmed the important effect of circumferential deformations on WSS estimations (Bertram 2009; Reymond et al. 2013).

From this point of view, the FSI model provides also more precise WSS values, resulting more reliable for a direct comparison with physiological WSS. In addition, the FSI-computed WSS (maximum of $3.960 \pm 0.082$ dyne/ $\mathrm{cm}^{2}$, minimum of $0.100 \pm 0.029$ dyne $/ \mathrm{cm}^{2}$ ) fully covered the range for EPCs differentiation towards endothelial phenotype (0.1-2.5 dyne $\left./ \mathrm{cm}^{2}\right)$ and the region of ECs preconditioning to increase cellular retention to the scaffold (Yamamoto et al. 2003; Uzarski et al. 2015).

Thus, our FSI model nicely describes the behavior of viscoelastic scaffolds in term of circumferential strains and WSS, under pulsatile perfusion. Use of experimentallyobtained material properties (Young's modulus and shear relaxation modulus in circumferential direction, and wall compliance) makes the model predictions scaffold-specific. However, the model does not take into account variability of the scaffold thickness along its length and inner diameter. Moreover, these results were related to the initial operation of the scaffold, since the effect of cell growth and the scaffold maturation were not considered. Modification of mechanical properties due to cell colonization and ECM production could also alter Young's modulus and the compliance of the scaffold, modifying the working pressure, circumferential strains and WSS. Pulsatile perfusion cultures will be performed, starting from the results of the FSI model and analyzing the variation of the mechanical properties due to cell remodeling. 
In conclusion, the developed FSI model represents a simple and quick method to evaluate a priori WSS and deformations able to ensure a physiological-like mechanical conditioning on the vascular cells-scaffold system, thus properly driving in vitro maturation. Therefore, besides saving experimental time and costs, the FSI model is pivotal for in silico initial evaluations of proper physiological mechanical stimulations of tissue-engineered viscoelastic scaffolds, based on scaffold mechanical properties experimentally obtained and a proper tuning of the bioreactor working setup, showing its innovative application in VTE field.

\section{Geolocation information}

Politecnico di Milano, Milan, Italy.

\section{Disclosure statement}

No potential conflict of interest was reported by the authors.

\section{References}

Alwan A, Armstrong T, Cowan M, Riley L, Alisalad A, Bae E, Bull F, Commar A, Dan L, Danaei G, et al. 2011. Global status report on noncommunicable diseases, World Health Organization. Available from: http://www.who.int/nmh/ publications/ncd_profiles2011/en/

Bavo AM, Rocatello G, Iannaccone F, Degroote J, Vierendeels J, Segers P. 2016. Fluid-structure interaction simulation of prosthetic aortic valves: comparison between immersed boundary and arbitrary Lagrangian-Eulerian techniques for the mesh representation. PLOS One. 11:e0154517.

Bertram CD. 2009. Fluid flow in distensible vessels. Clin Exp Pharmacol Physiol. 36:206-216.

Bilodeau K, Couet F, Boccafoschi F, Mantovani D. 2005. Design of a perfusion bioreactor specific to the regeneration of vascular tissues under mechanical stresses. Artif Organs. 29:906-912.

Boccafoschi F, Bosetti M, Mosca C, Mantovani D, Cannas M. 2012. The role of shear stress on mechanically stimulated engineered vascular substitutes: influence on mechanical and biological properties. J Tissue Eng Regen Med. 6:60-67.

Couet F, Mantovani D. 2012. Perspectives on the advanced control of bioreactors for functional vascular tissue engineering in vitro. Expert Rev Med Devices. 9:233-239.

Couet F, Meghezi S, Mantovani D. 2012. Fetal development, mechanobiology and optimal control processes can improve vascular tissue regeneration in bioreactors: an integrative review. Med Eng Phys. 34:269-278.

Cui Y, Wang X, Liu Z. 2010. Prediction the stress and strain field acting on the surface of engineered cells seeded in the $3 \mathrm{D}$ bioreactor. Second International Conference on Computer Engineering and Applications 2010. doi: 10.1109/ICCEA.2010.115

Dermenoudis S, Missirlis Y. 2010. Design of a novel rotating wall bioreactor for the in vitro simulation of the mechanical environment of the endothelial function. J Biomech. 43:1426-1431.
Fung YC. 1993. Biomechanics: mechanical properties of living tissues. New York, NY: Springer.

Gong X, Liu H, Ding X, Liu M, Li X, Zheng L, Jia X, Zhou G, Zou Y, Li J, et al. 2014. Physiological pulsatile flow culture conditions to generate functional endothelium on a sulfated silk fibroin nanofibrous scaffold. Biomaterials. 35:47824791.

Hahn MS, McHale MK, Wang E, Schmedlen RH, West JL. 2007. Physiologic pulsatile flow bioreactor conditioning of poly(ethylene glycol)-based tissue engineered vascular grafts. Ann Biomed Eng. 35:190-200.

Huang AH, Niklason LE. 2014. Engineering of arteries in vitro. Cell Mol Life Sci. 71:2103-2118.

Jeong SI, Kim SY, Cho SK, Chong MS, Kim KS, Kim H, Lee SB, Lee YM. 2007. Tissue-engineered vascular grafts composed of marine collagen and PLGA fibers using pulsatile perfusion bioreactors. Biomaterials. 28:1115-1122.

Kanyanta V, Ivankovic A, Karac A. 2009. Validation of a fluidstructure interaction numerical model for predicting flow transients in arteries. J Biomech. 42:1705-1712.

Kelly SC, O’Rourke MJ. 2010. A two-system, single-analysis, fluid-structure interaction technique for modelling abdominal aortic aneurysms. Proc Inst Mech Eng $\mathrm{H}$. 224:955-969.

Kona S, Chellamuthu P, Xu H, Hills SR, Nguyen KT. 2009. Effects of cyclic strain and growth factors on vascular smooth muscle cell responses. Open Biomed Eng J. 3:2838.

Lesman A, Blinder Y, Levenberg S. 2010. Modeling of flowinduced shear stress applied on 3D cellular scaffolds: implications for vascular tissue engineering. Biotechnol Bioeng. 105:645-654.

Montevecchi F, Redaelli A, 2007. Biomeccanica: analisi multiscala di tessuti biologici, p. 392.

Park YR, Kim SJ, Kim SJ, Kim JS, Kang HS, Kim GB. 2013. A study on hemodynamic characteristics at the stenosed blood vessel using computational fluid dynamics simulations. J Biomed Nanotechnol. 9:1137-1145.

Patrachari AR, Podichetty JT, Madihally SV. 2012. Application of computational fluid dynamics in tissue engineering. J Biosci Bioeng. 114:123-132.

Pellegata AF, Asnaghi MA, Stefani I, Maestroni A, Maestroni S, Dominioni T, Zonta S, Zerbini G, Mantero S. 2013. Detergent-enzymatic decellularization of swine blood vessels: insight on mechanical properties for vascular tissue engineering. Biomed Res Int. 2013:918753.

Raimondi MT, Boschetti F, Falcone L, Fiore GB, Remuzzi A, Marinoni E, Marazzi M, Pietrabissa R. 2002. Mechanobiology of engineered cartilage cultured under a quantified fluiddynamic environment. Biomech Model Mechanobiol. 1:6982.

Reymond P, Crosetto P, Deparis S, Quarteroni A, Stergiopulos N. 2013. Physiological simulation of blood flow in the aorta: comparison of hemodynamic indices as predicted by $3-\mathrm{D}$ FSI, 3-D rigid wall and 1-D models. Med Eng Phys. 35:784791.

Schmidt JB, Tranquillo RT. 2015. Cyclic stretch and perfusion bioreactor for conditioning large diameter engineered tissue tubes. Ann Biomed Eng. 44:1785-1797.

Silver FH, Horvath I, Foran DJ. 2001. Viscoelasticity of the vessel wall: the role of collagen and elastic fibers. Crit Rev Biomed Eng. 29:279-302. 
Sokolis DP, Kefaloyannis EM, Kouloukoussa M, Marinos E, Boudoulas H, Karayannacos PE. 2006. A structural basis for the aortic stress-strain relation in uniaxial tension. J Biomech. 39:1651-1662.

Stoitsis J, Golemati S, Bastouni E, Nikita KS. 2007. A mathematical model of the mechanical deformation of the carotid artery wall and its application to clinical data. Conf Proc IEEE Eng Med Biol Soc. 2007:2163-2166.

Uzarski JS, Cores J, McFetridge PS. 2015. Physiologically modeled pulse dynamics to improve function in in vitroendothelialized small-diameter vascular grafts. Tissue Eng Part C Methods. 21:1125-1134.

Wang Z, Wood NB, Xu XY. 2015. A viscoelastic fluid-structure interaction model for carotid arteries under pulsatile flow. Int J Numer Method Biomed Eng. 31:e02709.

WHO. 2014. World Health Statistics 2014. Available from: http://www.who.int/gho/publications/world_health_ statistics/2014/en/ and http://www.who.int/mediacentre/ factsheets/fs310/en

Schmedlen RH, Elbjeirami WM, Gobin AS, West JL. 2003. Tissue engineered small-diameter vascular grafts. Clin Plast Surg. 30:507-517.

Tresoldi C, Pellegata AF, Mantero S. 2015. Cells and stimuli in small-caliber blood vessel tissue engineering. Regen Med. 10:505-527.
Rotenberg MY, Ruvinov E, Armoza A, Cohen S. 2012. A multishear perfusion bioreactor for investigating shear stress effects in endothelial cell constructs. Lab Chip. 12:26962703.

Seliktar D, Black RA, Vito RP, Nerem RM. 2000. Dynamic mechanical conditioning of collagen-gel blood vessel constructs induces remodeling in vitro. Ann Biomed Eng. 28:351-362.

Song Y, Wennink JW, Kamphuis MM, Sterk LM, Vermes I, Poot AA, Feijen J, Grijpma DW. 2011. Dynamic culturing of smooth muscle cells in tubular poly(trimethylene carbonate) scaffolds for vascular tissue engineering. Tissue Eng Part A. 17:381-387.

Yamamoto K, Takahashi T, Asahara T, Ohura N, Sokabe T, Kamiya A, Ando J. 2003. Proliferation, differentiation, and tube formation by endothelial progenitor cells in response to shear stress. J Appl Physiol. 95:2081-2088.

Zhang X, Wang X, Keshav V, Wang X, Johanas JT, Leisk GG, Kaplan DL. 2009. Dynamic culture conditions to generate silk-based tissue-engineered vascular grafts. Biomaterials. 30:3213-3223.

Zahedmanesh H, Lally C. 2012. A multiscale mechanobiological modelling framework using agent based models finite element analysis application to vascular tissue engineering. Biomech Model Mechanobiol. 11:363-377. 